\title{
In situ functionalization of poly(hydroxyethyl methacrylate) cryogels with oligopeptides via $\beta$ - cyclodextrin-adamantane complexation for studying cell-instructive peptide environment
}

Thai Duong Luonga ${ }^{a \pm}$ Mohamed Zoughaib ${ }^{a \pm}$, Ruslan Garifullin $^{a, b, *}$, Svetlana Kuznetsova ${ }^{a}$, Mustafa O. Guler ${ }^{\mathrm{c}}$, Timur I. Abdullin ${ }^{\mathrm{a}, *}$

anstitute of Fundamental Medicine and Biology, Kazan Federal University, Kazan, Russia bInstitute of Materials Science and Nanotechnology, Bilkent University, Ankara, Turkey 'Pritzker School of Molecular Engineering, University of Chicago, Chicago, IL, USA

${ }^{ \pm}$These authors have equal contribution to this article.

*Corresponding authors:

T.Abdullin (tabdulli@gmail.com, timur.abdullin@kpfu.ru), R.Garifullin (rigarifullin@kpfu.ru) 
A
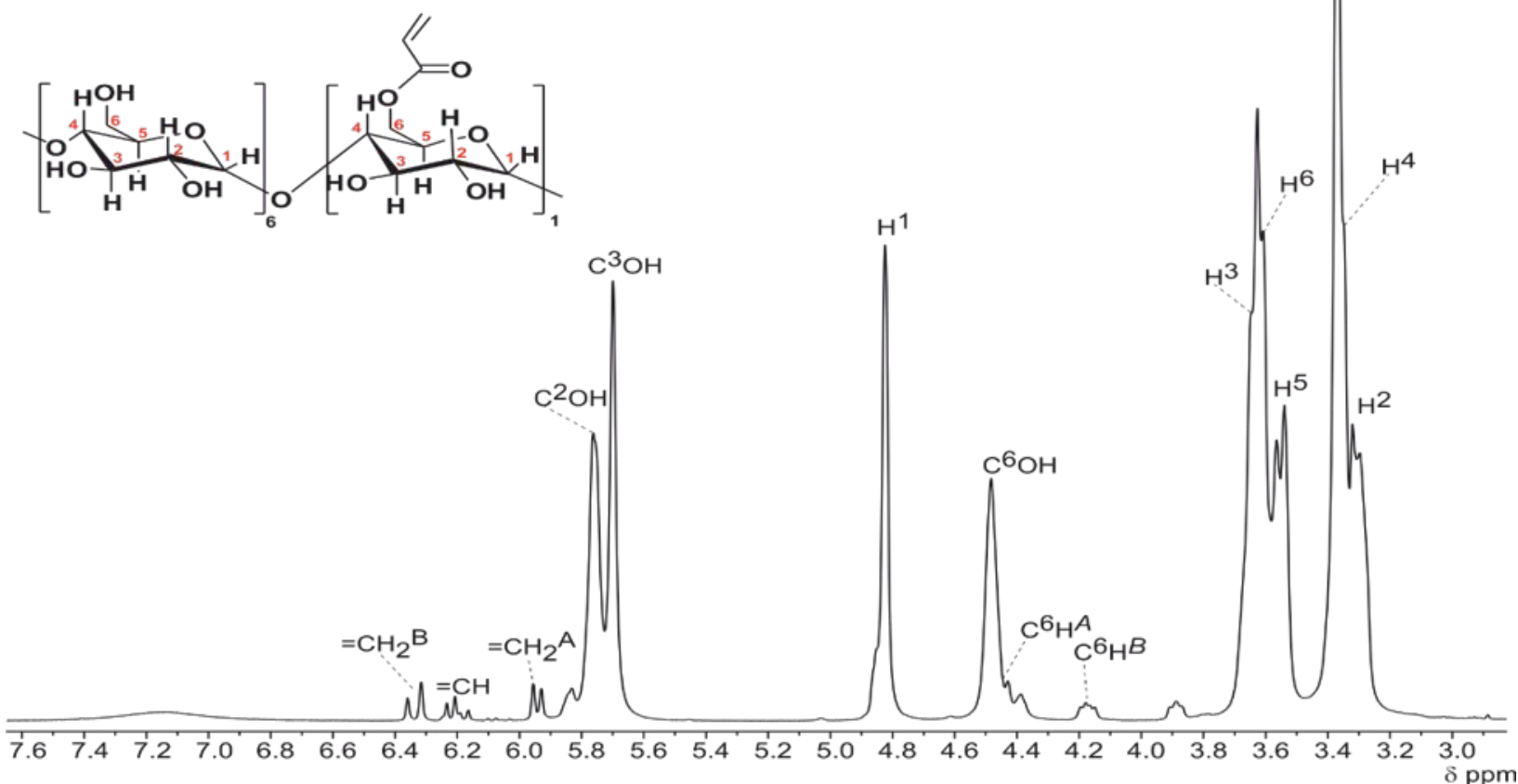

B

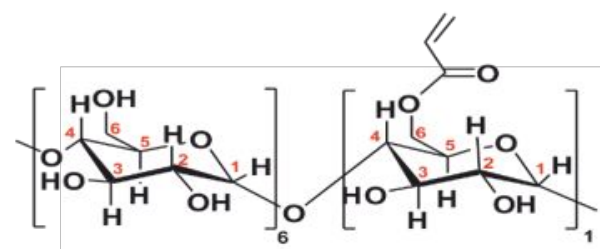

$c^{3} c^{2} c^{5}$

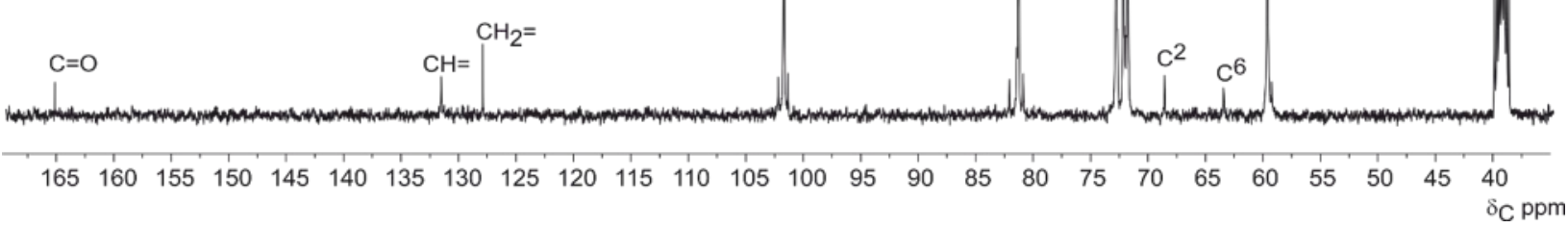



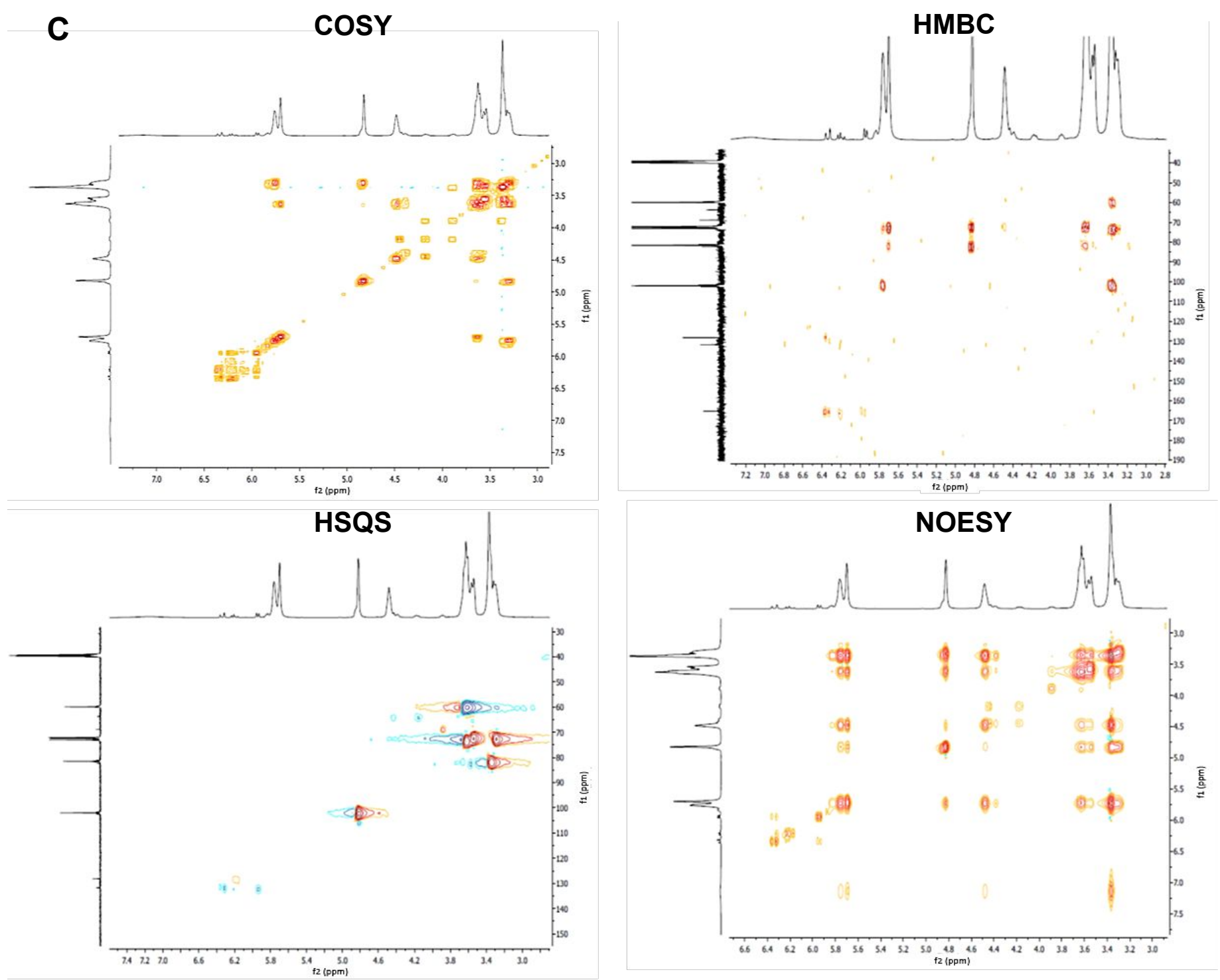

D

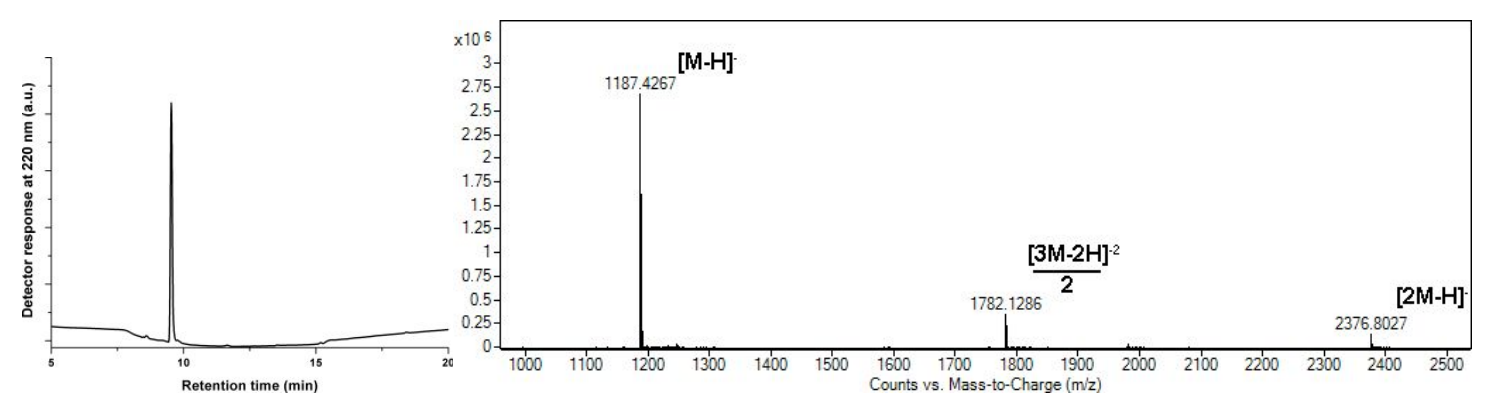

Figure S1. NMR and LC-MS spectra of acryloyl-CD. A. ${ }^{1} \mathrm{H}$ NMR spectrum (400 MHz, DMSO$d_{6}$ ). The peaks characteristic of the acrylate moiety appeared at chemical shifts range $\delta: 5.9-6.4$ ppm, attributed to $\mathrm{CH}_{2}=\mathrm{CH}$ of the methine and methylene protons in the vinyl group. B. ${ }^{13} \mathrm{C}$ NMR spectrum $\left(100.6 \mathrm{MHz}, \mathrm{DMSO}-\mathrm{d}_{6}\right)$. The peaks at 128,133 and $165 \mathrm{ppm}$ are associated, respectively, with $\mathrm{CH}_{2}=, \mathrm{CH}=$ and $\mathrm{C}=\mathrm{O}$ of the acrylate moiety. C. 2D (COSY, HMBC, HSQS, NOESY) NMR spectra. D. LC-MS spectra of acryloyl-CD. 


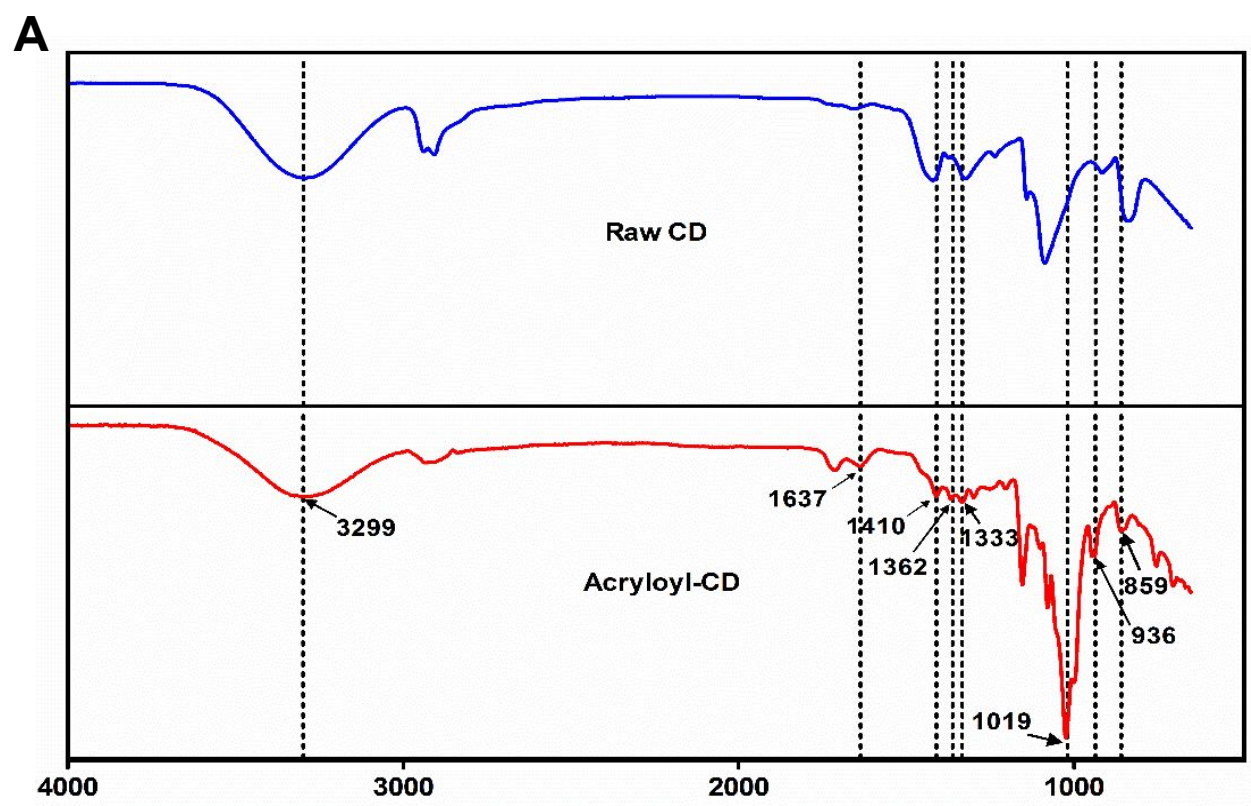

B

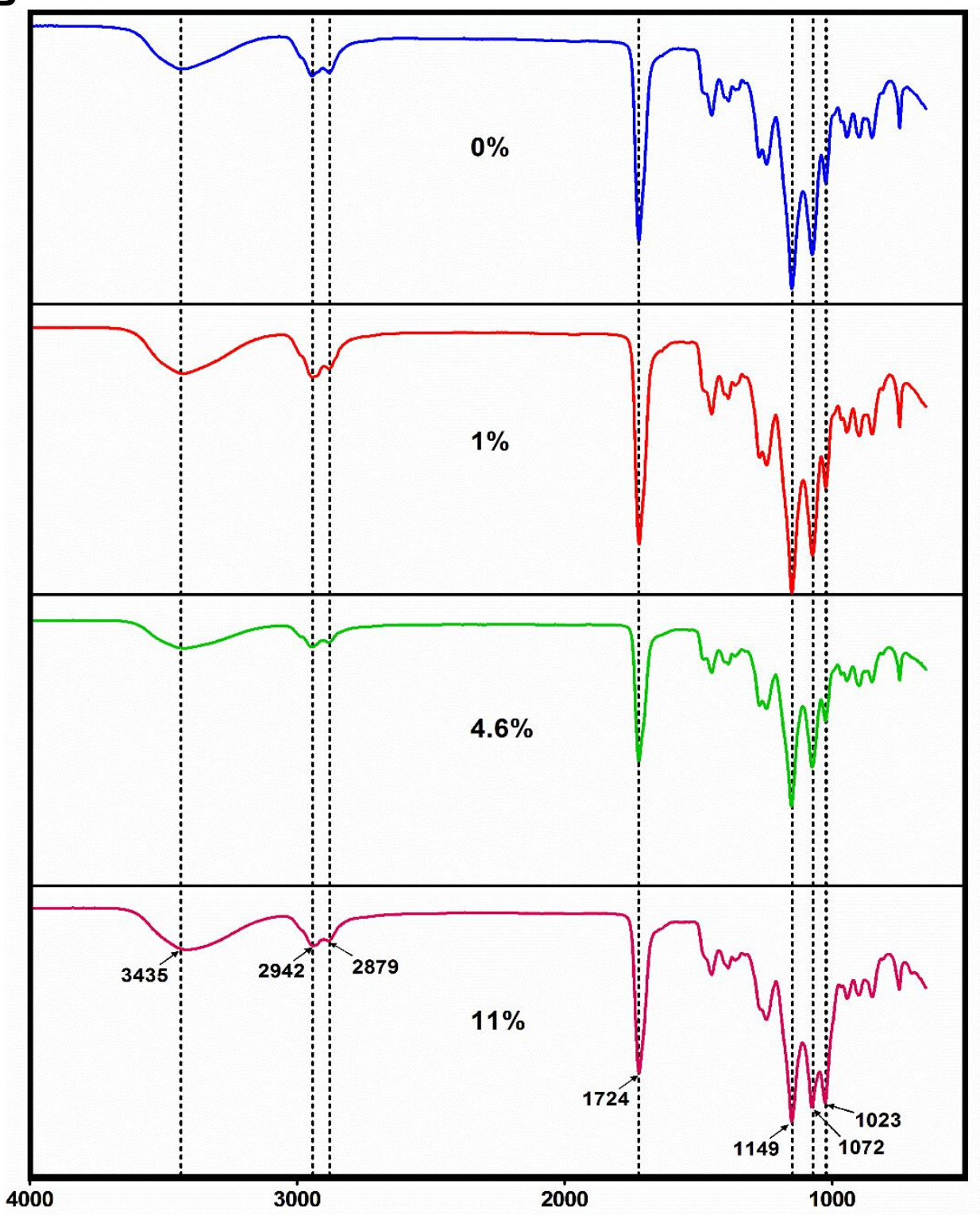

Figure S2. FTIR spectra of (A) $\beta$-cyclodextrin (CD)/acryloyl-CD, (B) pHEMA and CDmodified (pHEMA/CD) cryogels prepared with different $\mathrm{CD}$ concentrations (0-11\%). 

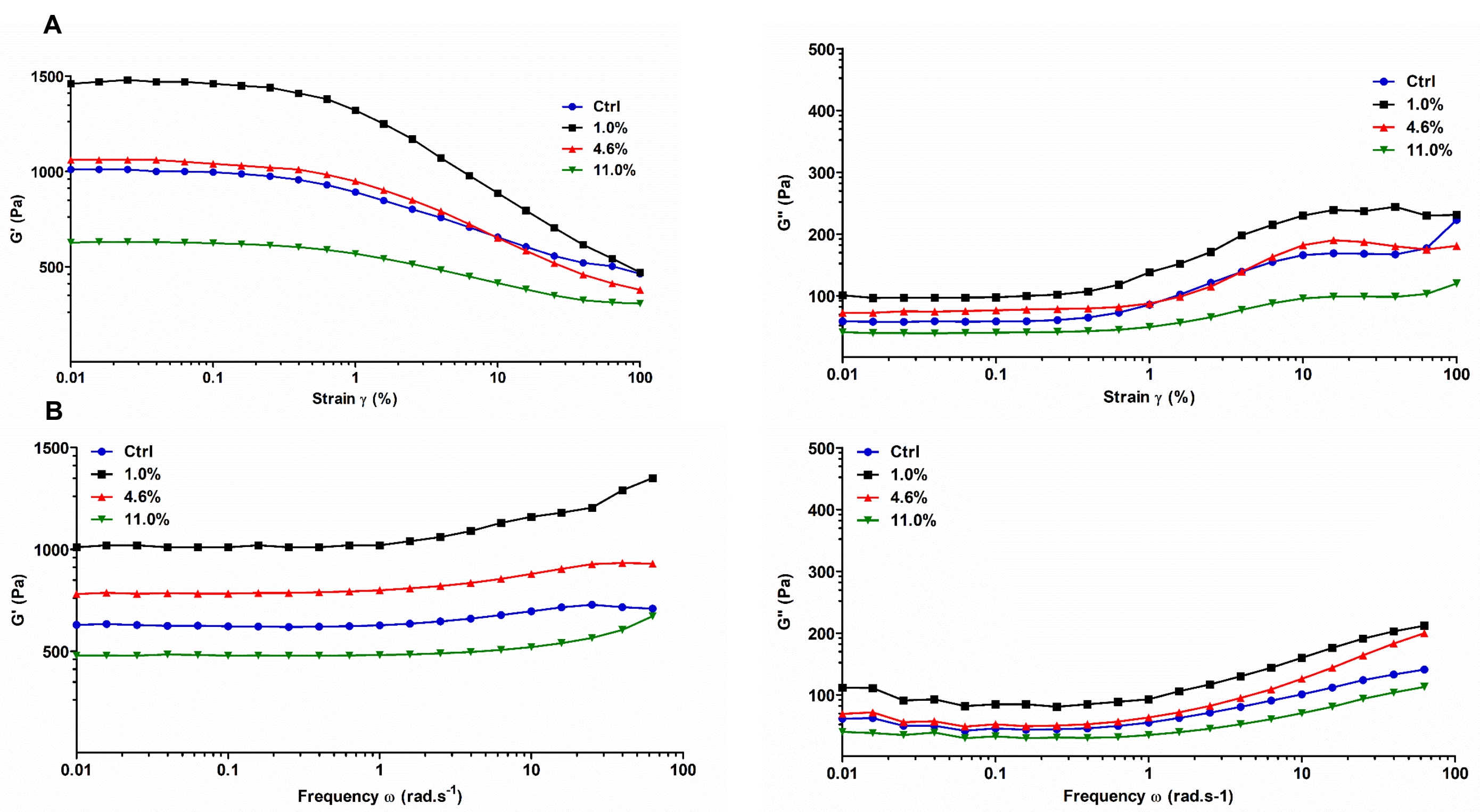

Figure S3. A. Strain amplitude sweep test of the CD-modified pHEMA cryogels. The storage $\mathrm{G}^{\prime}$ and loss G" moduli are shown as function of strain at angular frequency $\omega=10 \mathrm{rad} . \mathrm{s}^{-1}$. B. Frequency sweep test for the same samples. The measurement of frequency dependence of $\mathrm{G}^{\prime}$ and $\mathrm{G}^{\prime \prime}$ was performed within LVR at $\delta=1 \%$ strain deformation. 


\section{Ada-Ahx-GGRGD (1)}
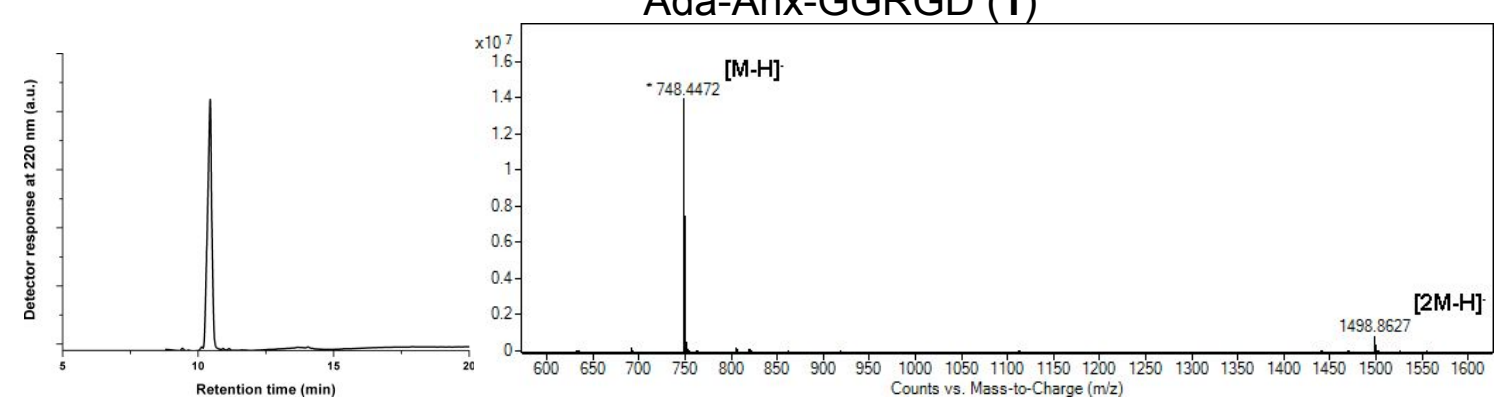

Ada-Ahx-GGGHK (2)
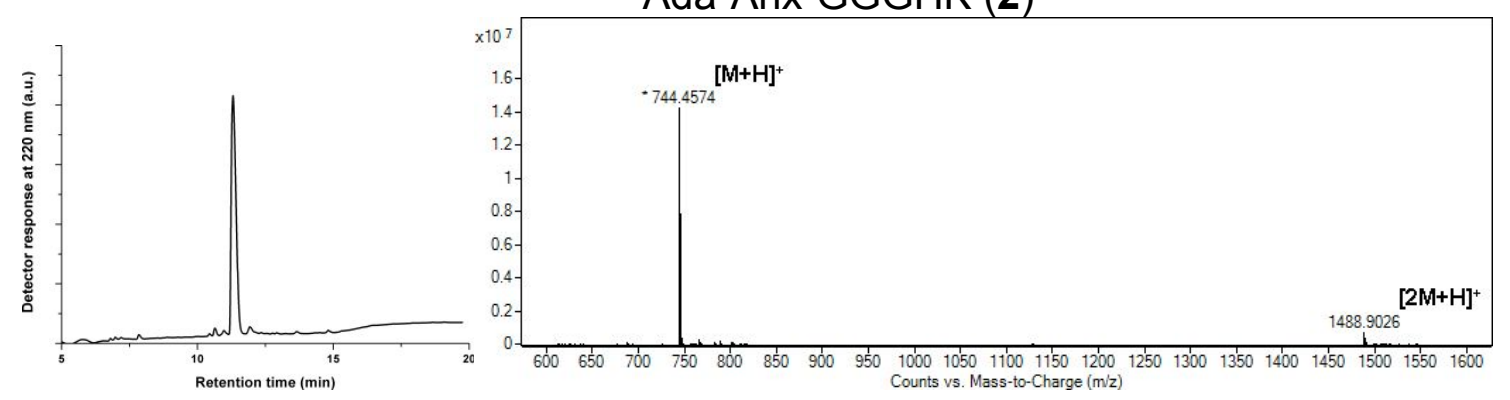

Ada-Ahx-GGK(pyrenebutyryl)GRGD (3)
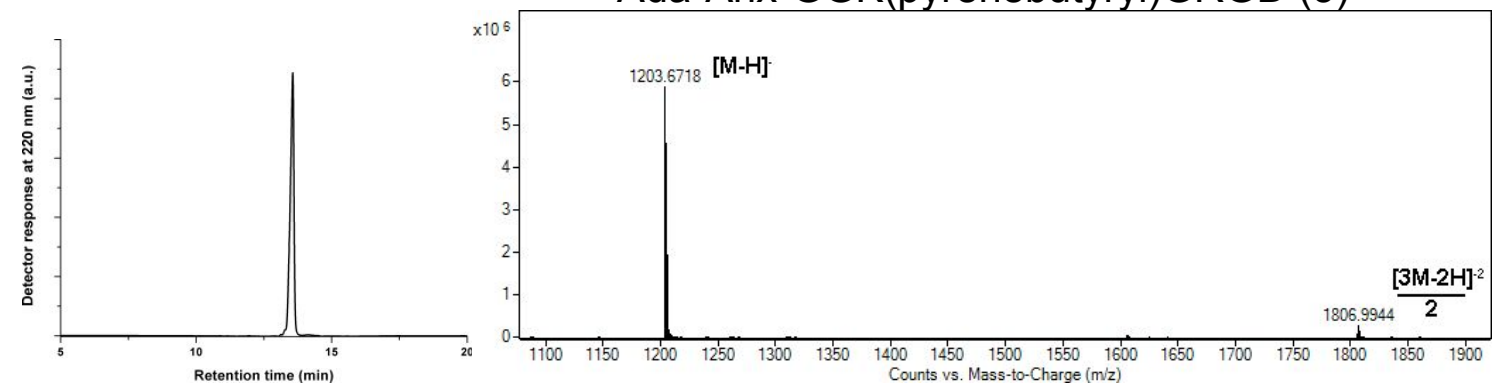

Ada-Ahx-GGK(dansylglycyl)GGHK (4)
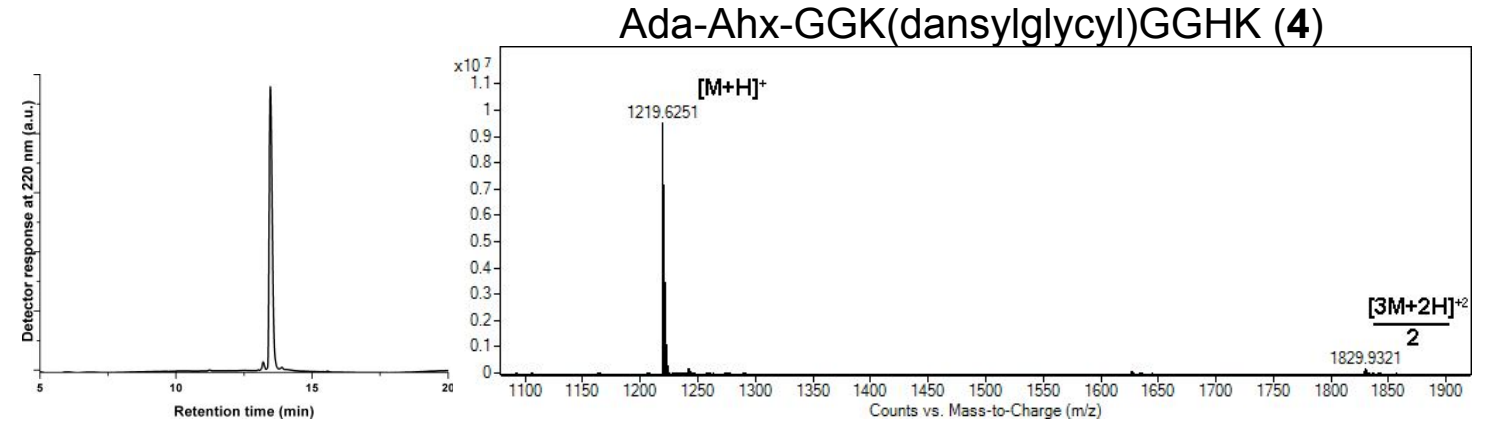

Figure S4. LC-MS spectra of Ada-Ahx-GGRGD (1), Ada-Ahx-GGGHK (2), Ada-AhxGGK(pyrenebutyryl)GRGD (3) and Ada-Ahx-GGK(dansylglycyl)GGHK (4) peptides. 


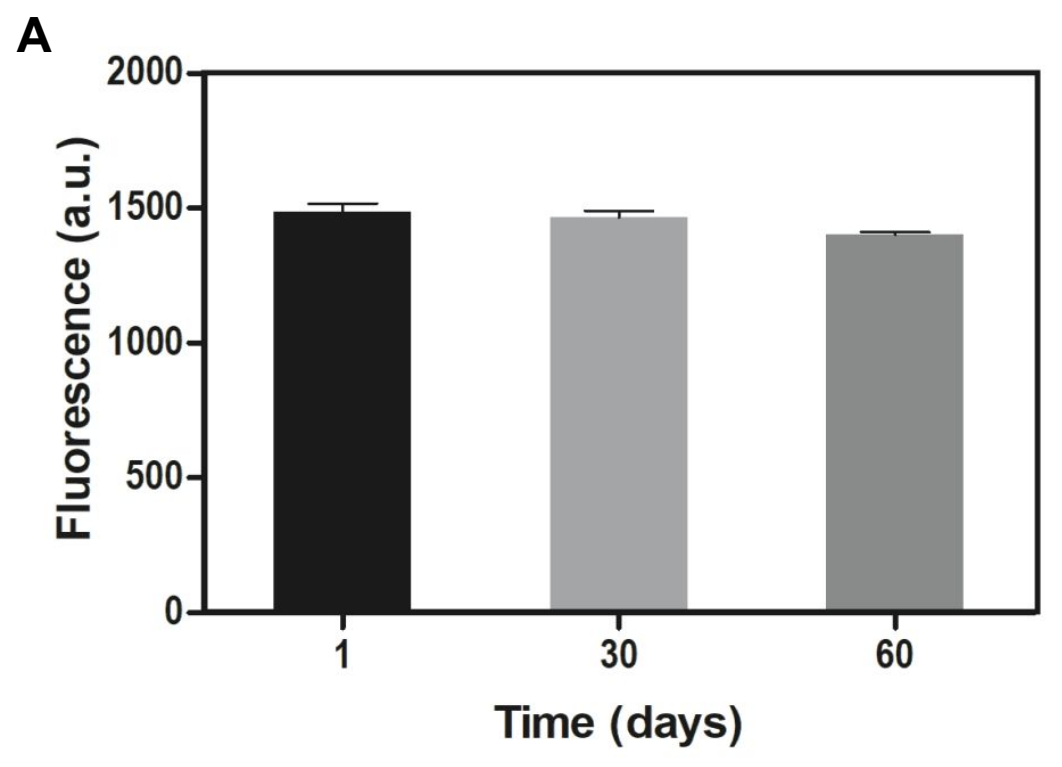

B

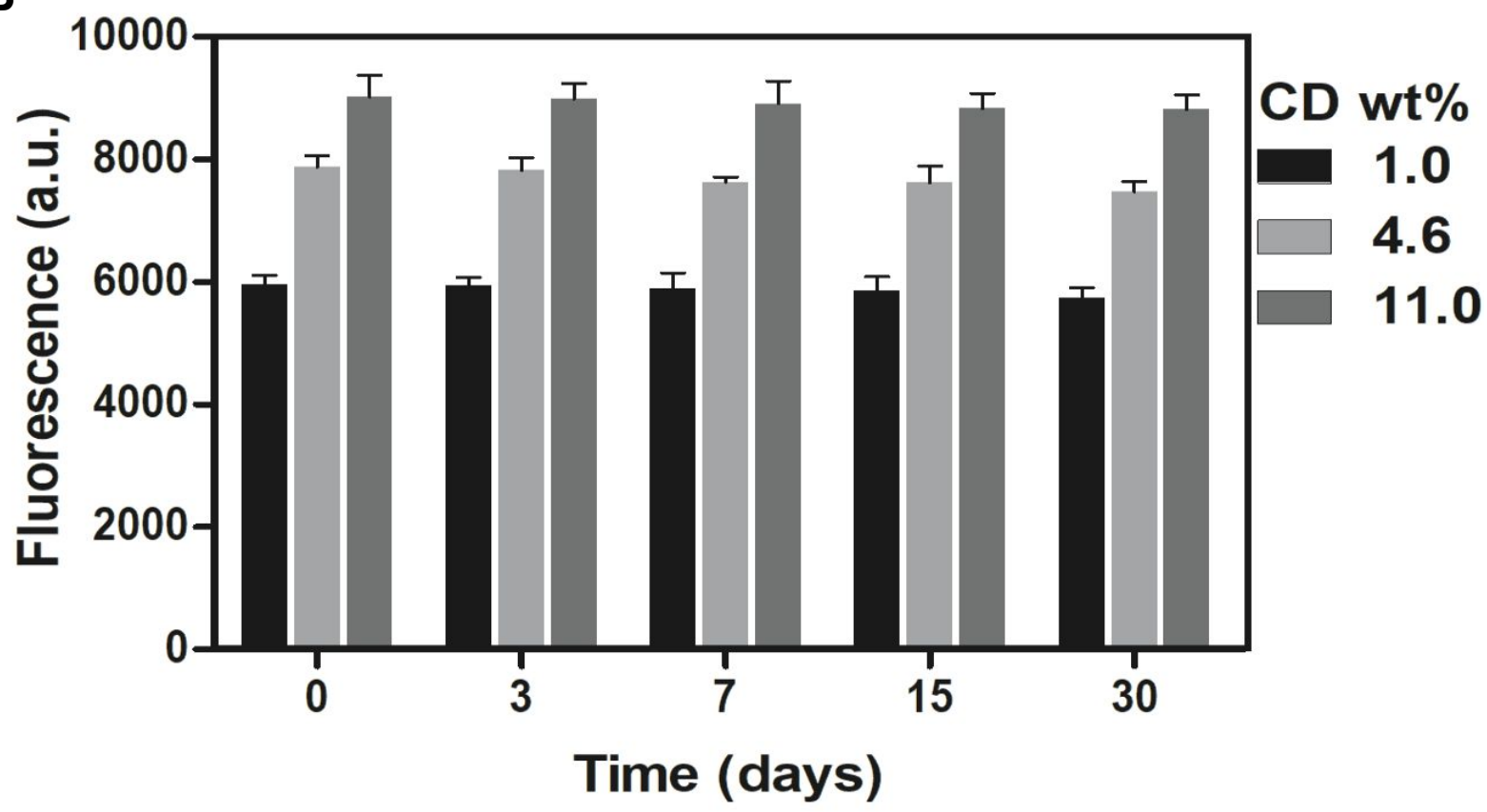

Figure S5. A. Mean fluorescence intensity of modified pHEMA cryogel (4.6\% CD) with affinity bound TBO; the material was stored during 60 days followed by staining with TBO. B. Mean fluorescence intensity of CD-modified pHEMA cryogels with affinity bound Ada-AhxGGK(pyrenebutyryl)GRGD peptide; the materials functionalization with the peptide was followed by storage during 30 days. 